\title{
Evaluation of Parkinson Disease and Alzheimer Disease with the Use of Neuromelanin MR Imaging and ${ }^{123}$ I-Metaiodobenzylguanidine Scintigraphy
}

\author{
F. Miyoshi, T. Ogawa, S.-i. Kitao, M. Kitayama, Y. Shinohara, M. Takasugi, S. Fujii, and T. Kaminou
}

\begin{abstract}
BACKGROUND AND PURPOSE: Progressive changes in the substantia nigra pars compacta and locus ceruleus of patients with Parkinson disease and Alzheimer disease visualized by neuromelanin MRI and cardiac postganglionic sympathetic nerve function on ${ }^{123}$ I-metaiodobenzylguanidine scintigraphy have not been fully evaluated. We compared the diagnostic value of these modalities among patients with early Parkinson disease, late Parkinson disease, and Alzheimer disease.
\end{abstract}

MATERIALS AND METHODS: We compared contrast ratios of signal intensity in medial and lateral regions of the substantia nigra pars compacta and locus ceruleus with those of the tegmentum of the midbrain and the pons, respectively, by use of neuromelanin MRI in patients with early Parkinson disease $(n=13)$, late Parkinson disease $(n=31)$, Alzheimer disease $(n=6)$, and age-matched healthy control subjects $(n=20)$. We calculated heart-to-mediastinum ratios on ${ }^{123}$ I-metaiodobenzylguanidine scintigrams after setting regions of interest on the left cardiac ventricle and upper mediastinum.

RESULTS: The signal intensity of the lateral substantia nigra pars compacta on neuromelanin MRI was significantly reduced in early and late Parkinson disease, and that of the medial substantia nigra pars compacta was gradually and stage-dependently reduced in Parkinson disease. The signal intensity of the locus ceruleus was obviously reduced in late Parkinson disease. Signal reduction was not significant in the substantia nigra pars compacta and locus ceruleus of patients with Alzheimer disease. The heart-to-mediastinum ratio on ${ }^{123}$ metaiodobenzylguanidine scintigrams was stage-dependently reduced in Parkinson disease and normal in Alzheimer disease. The signal intensity ratios in substantia nigra pars compacta and locus ceruleus on neuromelanin MRI positively correlated with the heart-tomediastinum ratio on ${ }^{123}$ I-metaiodobenzylguanidine scintigrams.

CONCLUSIONS: Both neuromelanin MRI and ${ }^{123}$-metaiodobenzylguanidine scintigraphy can help to evaluate disease progression in Parkinson disease and are useful for differentiating Parkinson disease from Alzheimer disease.

ABREVIATIONS: $\mathrm{LC}=$ locus ceruleus; $\mathrm{MIGB}={ }^{123}$-metaiodobenzylguanidine; $\mathrm{NmMRI}=$ neuromelanin MR imaging; $\mathrm{PD}=$ Parkinson disease; $\mathrm{SNc}=$ substantia nigra pars compacta

E arly Parkinson disease (PD) can easily be mistaken for any number of disorders, including other forms of parkinsonism, such as multiple system atrophy, progressive supranuclear palsy, corticobasal degeneration, and dementia with Lewy bodies. Other degenerative diseases, such as Alzheimer disease and primary lateral sclerosis, can also be mistaken for PD. ${ }^{1}$ Improving diagnostic accuracy is critical for the early differentiation of $\mathrm{PD}$ and other neurode-

Received December 2, 2012; accepted after revision January 25, 2013.

From the Division of Radiology (F.M., T.O., S.-i.K., Y.S., M.T., S.F., T.K.), Department of Pathophysiological Therapeutic Science; and Division of Neurology (M.K.), Department of Brain and Neurosciences, Faculty of Medicine, Tottori University, Yonago, Japan.

Please address correspondence to Toshihide Ogawa, MD, Division of Radiology, Department of Pathophysiological Therapeutic Science, Faculty of Medicine,

Tottori University, 36-1, Nishi-cho, Yonago 683-504, Japan; e-mail: ogawa@

med.tottori-u.ac.jp

http://dx.doi.org/10.3174/ajnr.A3567 generative types of parkinsonism because their prognoses are very different and the choice of treatment strategy is extremely important.

Neuromelanin is a dark pigment that locates within certain catecholamine neurons of the human brain, such as the dopaminergic neurons of the substantia nigra pars compacta $(\mathrm{SNc})$ and the noradrenergic neurons of the locus ceruleus (LC). ${ }^{2}$ It is thought to be formed as a by-product of the catecholamine metabolism cascade through enzymatic and/or oxidative polymerization. ${ }^{2,3} \mathrm{PD}$ is characterized by the progressive loss of dopaminergic neurons that contain neuromelanin in the SNc and of noradrenergic neurons in the LC. Several pathologic studies have shown the selective loss of ventral intermediate and lateral cell groups of the SNc in PD. ${ }^{4-6}$ Noradrenergic neurons are also lost in the LC of patients with Alzheimer disease. ${ }^{7}$

It is reported that neuromelanin MRI (NmMRI) could visualize decreased signal intensity in regions that reflect the loss of 


\begin{tabular}{lcccc}
\hline & $\begin{array}{c}\text { Early Parkinson } \\
\text { Disease }\end{array}$ & $\begin{array}{c}\text { Late Parkinson } \\
\text { Disease }\end{array}$ & $\begin{array}{c}\text { Alzheimer } \\
\text { Disease }\end{array}$ & Control \\
\hline Patients, $n$ & 13 & 31 & 6 & 20 \\
Hoehn and Yahr stage & $\mathrm{I}: 3, \mathrm{Il}: 10$ & $\mathrm{III}: 16, \mathrm{IV}: 12, \mathrm{~V}: 3$ & $/$ \\
Male/female & $5 / 8$ & $14 / 17$ & $3 / 3$ & $5 / 15$ \\
Age, $y$, mean \pm SD & $68.3 \pm 5.88$ & $71.8 \pm 8.95$ & $75.7 \pm 9.52$ & $74.8 \pm 5.41$ \\
Duration, $y$, mean \pm SD & $4.30 \pm 5.37$ & $9.48 \pm 6.86$ & $2.5 \pm 3$ & $/$ \\
Hasegawa Dementia Scale-Revised, mean \pm SD & $/$ & $/$ & $12.8 \pm 4.76$ \\
\hline
\end{tabular}

Note:-Age does not differ between patient and control groups ( $P=.082$; 1-way analysis of variance)

neurons containing neuromelanin ${ }^{8,9}$ and the signal intensity in the SNc and LC was greatly reduced on NmMRI from patients with PD. Several investigators subsequently reported that NmMRI could show a reduction in the contrast ratio and volume of the SNc. ${ }^{10,11}$

The physiologic analog of noradrenaline, ${ }^{123}$ I-metaiodobenzylguanidine (MIBG), traces uptake and transport both in noradrenaline presynaptic sympathetic nerve terminals and in subsequent vesicular storage. ${ }^{12}$ Postganglionic presynaptic cardiac sympathetic nerve endings can be noninvasively assessed by MIBG scintigraphy because a reduction of MIBG uptake indicates postganglionic sympathetic dysfunction. Cardiac MIBG uptake is reduced in patients with Lewy body diseases such as PD, as well as dementia with Lewy bodies, and MIBG scintigraphy can also help to differentiate PD from other types of parkinsonism. ${ }^{13,14}$

However, progressive changes in PD and Alzheimer disease have not been fully evaluated by NmMRI and MIBG scintigraphy. We determined the usefulness of these modalities for differentially diagnosing PD and Alzheimer disease by analyzing changes in signal intensity in the SNc and LC and MIBG uptake in the left cardiac ventricle and evaluated correlations between NmMRI and MIBG scintigraphy findings.

\section{MATERIALS AND METHODS \\ Patients}

We investigated patients who had been tentatively diagnosed with suspected PD and who were finally confirmed as having PD or Alzheimer disease between December 2008 and April 2012. Both MRI and MIBG scintigraphy acquired within 1 month were retrospectively evaluated. Probable PD and Alzheimer disease were diagnosed according to the criteria of the United Kingdom Brain Bank and National Institute of Neurologic and Communicative Disorders and Stroke and the Alzheimer Disease and Related Disorders Association, respectively. Patients with PD were assigned to groups with either early or late PD on the basis of Hoehn and Yahr staging. ${ }^{15}$ Early PD comprised stages I and II, and late PD comprised stages III, IV, and V.

Patients with symptomatic cerebrovascular diseases and other central nervous system disorders were strictly excluded from the NmMRI evaluation, and those with cardiac diseases, diabetes mellitus, and/or medications that can interfere with MIBG uptake were excluded from the MIBG scintigraphy assessment.

We finally enrolled 13 (male, $n=5$; female, $n=8$ ), 31 (male, $n=14$; female, $n=17$ ), and 6 (male and female, $n=3$ each) patients with early $\mathrm{PD}$, late $\mathrm{PD}$, and Alzheimer disease, respectively (Table 1) [ages (mean \pm SD with range) $68.3 \pm 5.88$ (59-85), $71.8 \pm$ 8.95 (59-83), and $75.7 \pm 9.52(58-84)$ years, respectively]. The mean ( \pm SD with range) duration of early PD, late PD, and Alzhei- mer disease was $4.30 \pm 5.37(0-9), 9.48 \pm 6.86(2-27)$, and $2.5 \pm$ 3.00 (1-3) years, respectively. We used the Hasegawa Dementia Scale-Revised ${ }^{16}$ to determine cognitive impairment or dementia, which is similar to the Mini-Mental State Examination and has a total score of 30. Hasegawa Dementia Scale-Revised scores in patients with Alzheimer disease ranged from 6-17 (mean \pm SD, $12.8 \pm 4.76$ ).

Twenty age-matched patients (male, $n=5$; female, $n=15$ ) [ages (mean \pm SD with range) $74.8 \pm 5.41$ (64-87) years] without a history of motor or cognitive impairment and significant abnormalities on brain MRI during the same period served as a control group of NmMRI. However, we did not obtain MIBG scintigraphic data from the control group during this period.

Our institutional review board approved the study, and written informed consent was waived.

\section{Image Acquisition}

All MR images were acquired by means of a clinical 3T MR scanner (Signa Excite HD, GE Healthcare, Milwaukee, Wisconsin). Axial images were acquired parallel to the anterior/posterior commissure line. T1-weighted fast spin-echo sequences were applied to NmMRI with the following parameters: TR/TE, 600/13 ms; echo-train length, 2 ; section thickness, $2.5 \mathrm{~mm}$ with 1 - $\mathrm{mm}$ intersection gaps; matrix size, $512 \times 512$; FOV, $220 \mathrm{~mm}$; acquisition time, 12 minutes. The scan covered the area from the upper border of the midbrain to the inferior border of the pons. We excluded other coexisting central nervous system disorders by use of axial T1- and T2-weighted images, fluid-attenuated inversion recovery images, and diffusion-weighted images of the entire brain according to the following standard protocol for adult brain imaging at our hospital: T1-weighted spin-echo sequence, TR/TE, 600/15 ms; section thickness, 5 mm; FOV 220 mm; matrix $512 \times$ 512; T2-weighted fast spin-echo sequence, TR/TE, 4000/90 ms; section thickness, $5 \mathrm{~mm}$; FOV $220 \mathrm{~mm}$; matrix $512 \times 512$; fluid attenuated inversion recovery sequence, TR/TE/IR, 4000/90/20; section thickness, $5 \mathrm{~mm}$; FOV $220 \mathrm{~mm}$; matrix $512 \times 512$; and diffusion-weighted imaging sequence, TR/TE, 4000/90 ms; section thickness, $5 \mathrm{~mm}$; FOV $220 \mathrm{~mm}$; matrix $512 \times 512$; maximum b factor, $1000 \mathrm{~mm}^{2} / \mathrm{s}$.

The patients received an intravenous injection of $111 \mathrm{MBq}$ of ${ }^{123} \mathrm{I}-\mathrm{MIBG}$, and static planar images of the chest were acquired 30 minutes later for 4 minutes in a $256 \times 256$ matrix by use of a dualhead gamma camera with a large field of view and a low-energy, highresolution collimator (e.cam; Siemens, Erlangen, Germany). ${ }^{17}$

\section{Image Analysis}

Signal intensity was measured for quantitative NmMRI by setting regions of interest. We equally divided the SNc into medial and lateral regions at the level of the inferior colliculus and defined 


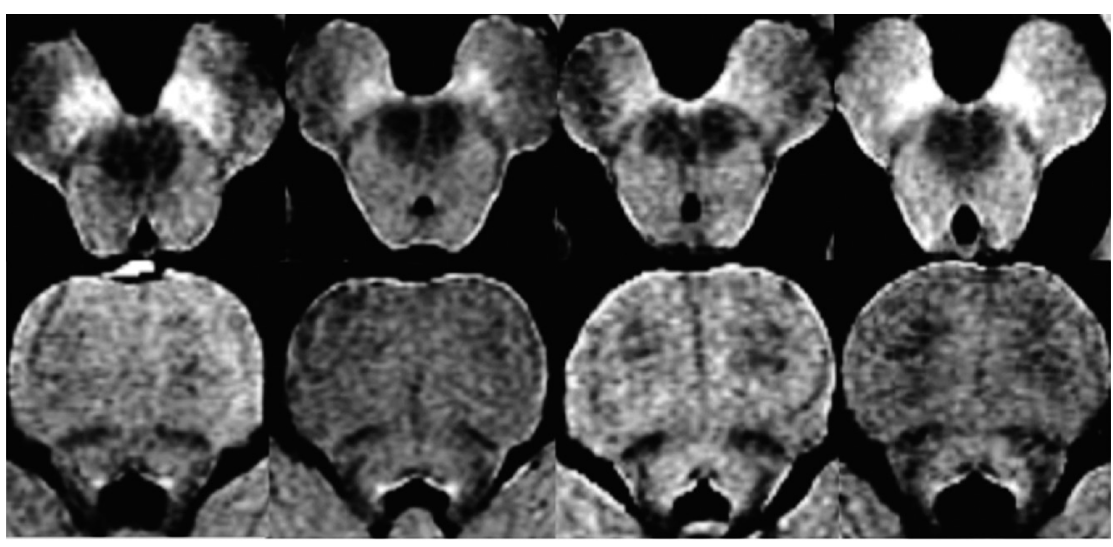

A

control

early PD

late PD

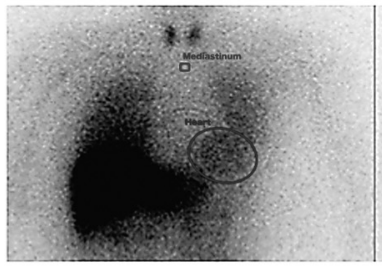

B early PD

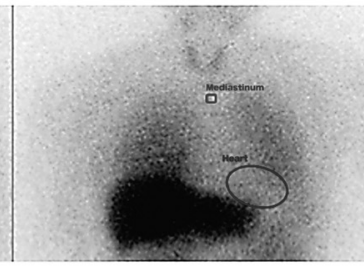

late PD

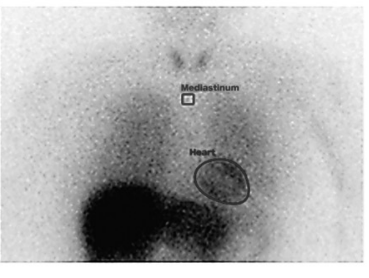

Alzheimer's disease
FIG 1. Findings of neuromelanin MRI and ${ }^{123}$-metaiodobenzylguanidine scintigrams of patients and control subjects. A, NmMRI: Signals are reduced in NmMRI of substantia nigra pars compacta and locus ceruleus according to Parkinson disease stage. B, MIBG scintigraphy: MIBG scintigrams show PD stage-dependent cardiac MIBG reduction.

ROIs in these areas that included high signal intensity on NmMRI. We also defined ROIs symmetrically in the ventral tegmentum located in the anterolateral areas of aqueduct as controls. Concerning the LC, we defined the ROIs in the anterolateral areas around the fourth ventricle at the level of upper pons and also defined ROIs symmetrically in the tegmentum located just behind the medial lemniscus as controls. The sizes of the region of interest were 8,2 , and $10 \mathrm{~mm}^{2}$ on the SNc, LC, and tegmentum of the midbrain and pons, respectively. We calculated the contrast ratios of the 3 bilateral portions by dividing their signal intensity by that of control areas such as the tegmentum of midbrain and pons.

An ROI was drawn manually over the whole heart on MIBG scintigrams to assess the global myocardial kinetics of MIBG. A second rectangular region of interest over the upper mediastinum served as a background reference region. The attenuation of the MIBG count in the heart and the mediastinum and heart-to-mediastinum count ratios were calculated for the images.

\section{Statistical Analysis}

Differences in contrast ratios between early PD, late PD, Alzheimer disease groups, and control subjects in the medial SNc, lateral SNc, and LC on NmMRI and between early PD, late PD, Alzheimer disease groups, and control subjects ${ }^{17}$ on MIBG scintigraphy were then statistically analyzed. The medial SNc, lateral SNc, and MIBG scintigrams were analyzed by means of a 1-way analysis of variance and the Bonferroni post hoc test, and the LC was analyzed by means of the Kruskal-Wallis and Dunn post hoc tests. The level of statistical significance was defined as $P<.05$ for all

\section{DISCUSSION}

Alzheimer's disease

tests. The contrast ratios of NmMRI and MIBG scintigram in early PD, late PD, and Alzheimer disease were analyzed by means of Spearman rank-order correlation coefficient test.

\section{RESULTS}

The signal intensity of the lateral SNc on NmMRI was reduced in early and late $\mathrm{PD}$, and that of the medial SNc was gradually and stage-dependently reduced in PD. The signal intensity of LC was obviously reduced in late PD. Signal reduction was not significant in the SNc and LC of patients with Alzheimer disease (Fig 1A). The heart-to-mediastinum ratio on MIBG scintigrams was stage-dependently reduced in PD and normal in Alzheimer disease (Fig 1B).

Quantitative NmMRI revealed smaller contrast ratios in the lateral SNc of patients with early PD than in that of control patients, and in that of patients with late $\mathrm{PD}$ than in that of patients with Alzheimer disease and control subjects $(P<$ .05) (Fig 2A). The contrast ratios of the medial SNc were smaller in patients with early PD than in control subjects and in patients with late $\mathrm{PD}$ than in those with early PD, Alzheimer disease, and control subjects $(P<.05)$ (Fig $2 B)$. Contrast ratios in the $\mathrm{LC}$ of patients with late $\mathrm{PD}$ were smaller than in control patients $(P<.05)$ (Fig $2 C)$. Signals were not significantly reduced in the SNc and LC on NmMRI of patients with Alzheimer disease compared with control subjects.

The heart-to-mediastinum count ratio on the MIBG scintigram was stage-dependently reduced in patients with $\mathrm{PD}(P<$ $.05)$ and normal (mean $\pm \mathrm{SD}, 2.15 \pm 0.15^{17}$ ) in those with Alzheimer disease (mean \pm SD, $2.19 \pm 0.40)($ Fig $2 D)$.

Signal intensity ratios in the SNc on NmMRI positively correlated with heart-to-mediastinum count ratios on MIBG scintigrams (Fig 3). Spearman rank-order correlation coefficients in the medial and lateral SNc were 0.358 and 0.398 , respectively, which reached statistical significance (Table 2).

Symmetrical spots of high signal intensity were visualized from around the anterolateral region to the floor of the fourth ventricle corresponding to the LC on axial NmMRI of the control group, and symmetrical bandlike high signal intensity was located around the posteromedial region of the cerebral peduncle corresponding to the SNc. Sasaki et $\mathrm{al}^{8}$ reported that their distribution obviously correlated with those of neuromelanin in gross specimens of the SNc and the LC, suggesting that the high signal intensity areas corresponded to neurons containing neuromelanin. We proved that NmMRI can accurately reflect the amount of neurons containing neuromelanin in the SNc, on the basis of a direct comparison of histopathologic and NmMRI findings of autopsied brains (unpublished data). 


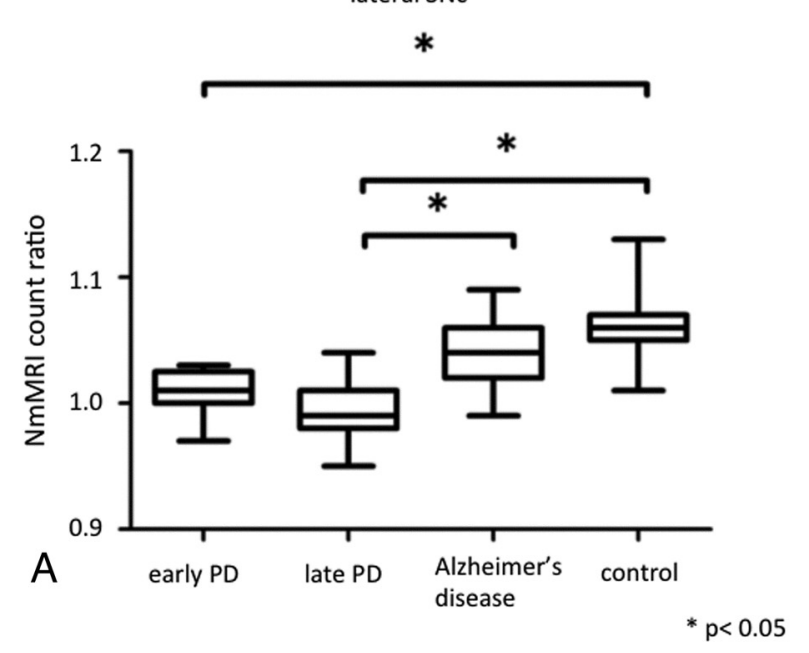

LC

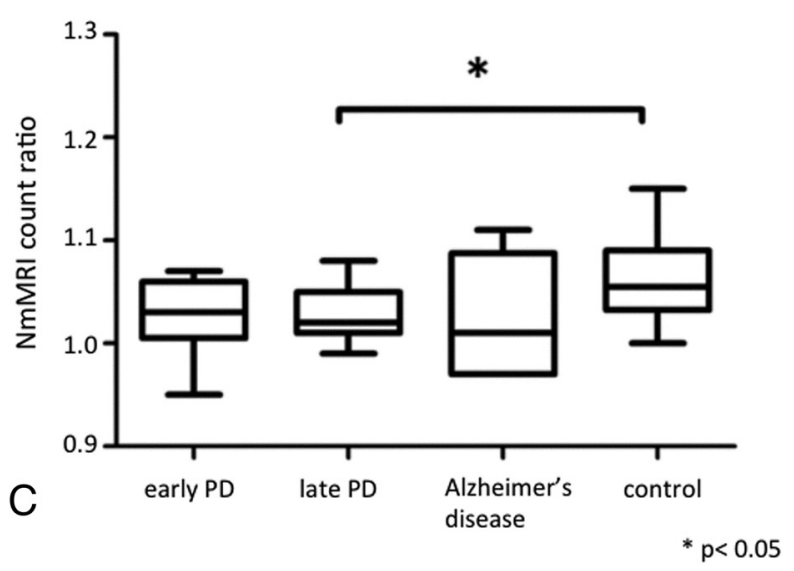

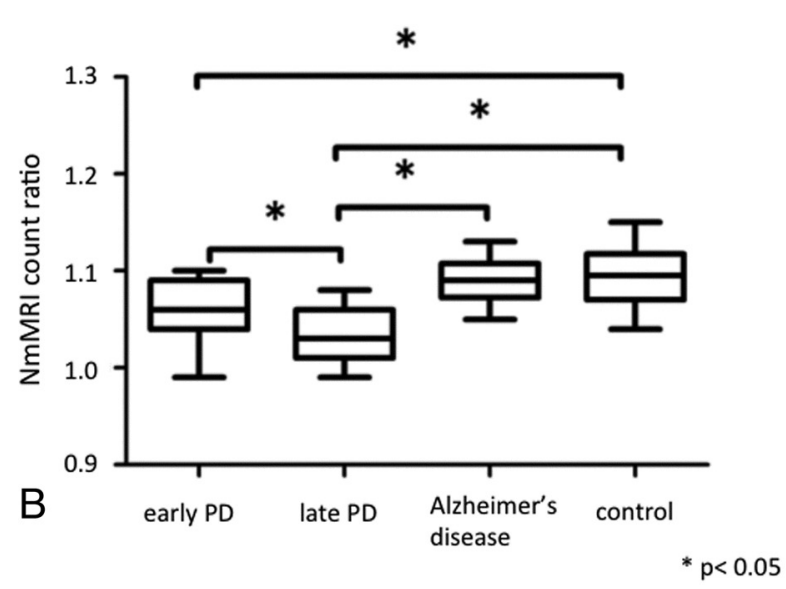

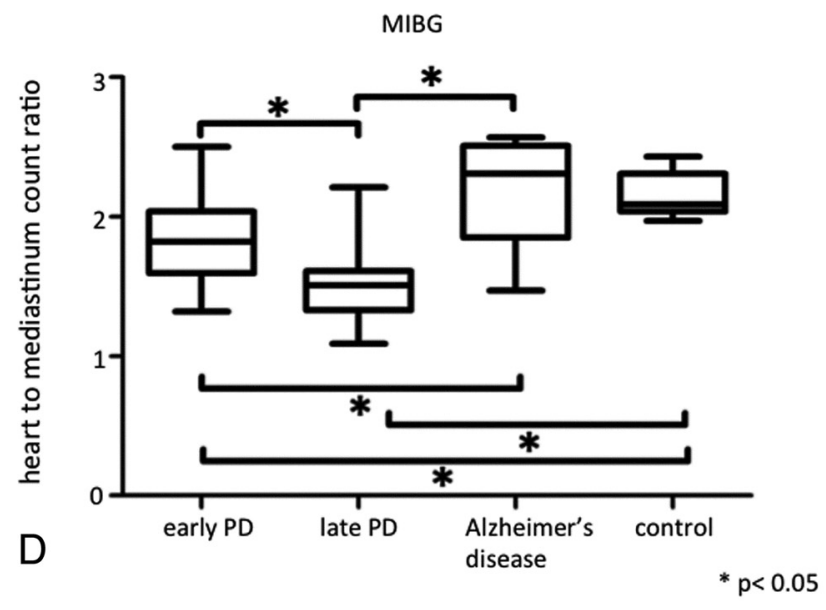

FIG 2. Box-and-whisker plots of signal intensity ratios on neuromelanin MRI and heart-to-mediastinum count ratios on ${ }^{123}$-metaiodobenzylguanidine scintigrams in patients with Parkinson disease, patients with Alzheimer disease, and control subjects. Horizontal bars in boxes show median values. $A$, Lateral substantia nigra pars compacta; $B$, medial SNc; $C$, locus ceruleus; $D$, MIBG. ${ }^{*}<.05$.

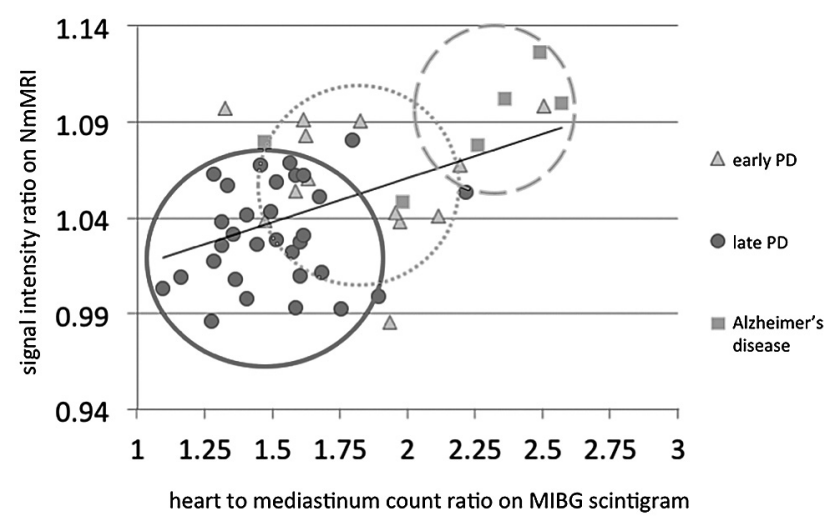

FIG 3. Correlation between signal intensity ratio in medial substantia nigra pars compacta on neuromelanin MRI image and heart-to-mediastinum count ratio on ${ }^{123}$-metaiodobenzylguanidine scintigram. $\rho=$ 0.3984 .

We found that the contrast ratio of the lateral SNc was significantly decreased in early and late PD and that of the medial SNc gradually and stage-dependently decreased in PD, which corresponded to the pathologic lesions. These findings indicate that
Table 2: Correlation between signal intensity ratio in medial SNc on NmMRI and heart-to-mediastinum count ratio on MIBG scintigraphy

\begin{tabular}{cccc}
\hline Spearman Coefficient Rate $(\boldsymbol{\rho})$ & Lateral SNc & Medial SNc & LC \\
\hline Heart-to-mediastinum count ratio & 0.358 & 0.398 & NS \\
\hline
\end{tabular}

$\mathrm{SNc}$ lesions extend from the lateral to the medial regions as PD progresses. Tanaka et $\mathrm{al}^{18}$ reported that a reduction in neuromelanin contrast started ventrolaterally and advanced medially in the substantia nigra on the basis of a visual assessment of NmMRI. However, the present study is the first effort to quantify medial and lateral SNc by use of NmMRI, to the best of our knowledge.

Braak et $\mathrm{al}^{19}$ proposed that $\alpha$-synuclein-immunopositive Lewy neuritis and the accumulation of Lewy bodies that are characteristic pathologic features of PD emerge from the inferior brain stem and ascend to the nuclear gray and cortical areas. However, some studies have also indicated that Lewy bodies and neural cell loss are irrelevant in some regions of the brain in patients with PD. Noradrenergic neurons are relatively well preserved in the LC of patients with early $\mathrm{PD}$, and they undergo different intracellular changes from the SNc. ${ }^{20}$

Reports indicate that the signal intensity of the LC on NmMRI 
is significantly reduced in $\mathrm{PD} .{ }^{8}$ We identified significant signal reductions in the $\mathrm{LC}$ of patients with late $\mathrm{PD}$, which suggests that lesions emerge in the LC during late PD. These findings are compatible with the findings of others indicating that the LC plays a compensatory role for the substantia nigra during early PD. ${ }^{21,22}$

Noradrenergic neurons containing neuromelanin in the LC are disrupted in Alzheimer disease, but the clinical or functional importance of such disruption remains unclear. ${ }^{7}$ Some reports suggest that a reduction in the number of noradrenergic neurons affects the accumulation of $\beta$-amyloid, inflammation, or microcirculation in the LC. ${ }^{23,24}$ Our NmMRI study could not identify a significant signal reduction in the LC of patients with Alzheimer disease. Busch et $\mathrm{al}^{25}$ reported that the LC begins to lose cells only during the later stages of Alzheimer disease. Therefore, we consider that this result can be attributed to the relatively short duration (2.5 years) of the disease and the small number of patients with Alzheimer disease in this study. Signals were significantly more reduced in the medial and lateral SNc of patients with late PD compared with Alzheimer disease, which is useful for differentiating these diseases.

The neurologic diseases with significant reduction in MIBG scintigraphy uptake include PD, dementia with Lewy bodies, and pure autonomic failure, and they are recognized as characteristic findings of Lewy-body diseases. On the other hand, heart-to-mediastinum count ratios are higher in Parkinson syndromes such as multiple system atrophy, progressive supranuclear palsy, and corticobasal degeneration than in PD. Therefore, MIBG scintigraphy is an important supportive tool for discerning Parkinson syndromes. ${ }^{26}$ Sympathetic nerves in patients with PD are generally disturbed, and Lewy bodies are found in sinoatrial nodal ganglia, the myocardium, and paravertebral ganglia. ${ }^{27,28} \mathrm{~A}$ pathologic investigation has revealed that sympathetic nerve fibers are significantly reduced in patients with PD and well preserved in those with Alzheimer disease. ${ }^{29}$ Although the heart-to-mediastinum count ratio is reduced on MIBG scintigrams of early PD, its relationship with progression remains controversial. ${ }^{29,30}$ In our present study, we found that the heart-to-mediastinum count ratio was significantly lower in late PD than early PD, indicating that signal reduction is stage-dependent. On the other hand, we could not exclude the effect of a possible relationship between heart-tomediastinum signal reduction and duration. The heart-to-mediastinum count ratio was well-preserved in patients with Alzheimer disease, which helped to differentiate it from late PD.

To the best of our knowledge, this is the first comparison of brain NmMRI and MIBG scintigraphy of PD and Alzheimer disease. We identified a weak correlation in the SNc, implying that the findings of both modalities can serve as indicators of progressive $\mathrm{PD}$, which involves both the central and peripheral autonomic nervous systems. Our results revealed that the autonomic nervous system gradually becomes disturbed over time.

The limitations of the present study are as follows. Because the study comprised very few patients with Alzheimer disease, further studies of a large population are required to validate our findings. Second, iron concentrations in the SNc that increase with age can mask signal alterations on NmMRI. ${ }^{3}$ Therefore, we could not exclude this effect when evaluating neuromelanin in elderly individuals. Third, the low spatial resolution of NmMRI did not allow 3D image acquisition. Therefore, measurement errors might have arisen due to partial volume effects particularly when assessing changes in the LC.

\section{CONCLUSIONS}

The SNc becomes disturbed from the lateral to the medial region on NmMRI of the SNc as PD progresses and the LC is also disturbed in late PD. The sympathetic nerves in the left cardiac ventricle are also disturbed in MIBG scintigrams of PD. Thus, NmMRI and MIBG scintigraphy can be helpful tools to evaluate PD progression and to differentiate PD from Alzheimer disease.

\section{ACKNOWLEDGMENTS}

We thank Eijirou Yamashita, PhD, of the Division of Clinical Radiology, Tottori University Hospital, for support with MR imaging.

\section{REFERENCES}

1. Pahwa R, Lyons KE. Early diagnosis of Parkinson's disease: recommendations from diagnostic clinical guidelines. Am J Manag Care 2010;16(Suppl):S94-99

2. Fedorow H, Tribl F, Halliday G, et al. Neuromelanin in human dopamine neurons; comparison with peripheral melanins and relevance to Parkinson's disease. Prog Neurobiol 2005;75:109-24

3. Zecca L, Tampellini D, Gerlach M, et al. Substantia nigra neuromelanin: structure, synthesis, and molecular behavior. Mol Pathol 2001;54:414-18

4. Damier P, Hirsch EC, Agid Y, et al. The substantia nigra of the human brain, II: patterns of loss of dopamine-containing neurons in Parkinson's disease. Brain 1999;122:1437-48

5. Fearnley JM, Lees AJ. Aging and Parkinson's disease: substantia nigra regional selectivity. Brain 1991;114:2283-301

6. Halliday GM, McRitchie DA, Cartwright HR, et al. Midbrain neuropathology in idiopathic Parkinson's disease and diffuse Lewy body disease. J Clin Neurosci 1996;3:52-60

7. Förstl H, Burns A, Luthert $P$, et al. Clinical and neuropathological correlates of depression in Alzheimer's disease. Psychol Med 1992;22:877-84

8. Sasaki M, Shibata E, Tohyama K, et al. Neuromelanin magnetic resonance imaging of locus ceruleus and substantia nigra in Parkinson's disease. NeuroReport 2006;17:1215-18

9. Mann DM, Yates PO. Possible role of neuromelanin in the pathogenesis of Parkinson's disease. Mech Ageing Dev 1983;21:193-203

10. Schwarz ST, Rittman T, Gontu V, et al. T1-weighted MRI shows stage-dependent substantia nigra signal loss in Parkinson's disease. Mov Disord 2011;26:1633-38

11. Kashihara K, Shinya T, Higaki F. Neuromelanin magnetic resonance imaging of nigral volume loss in patients with Parkinson's disease. J Clin Neurosci 2011;18:1093-96

12. Wieland DM, Brown LE, Rogers WL, et al. Myocardial imaging with a radioiodinated norepinephrine storage analog. I Nucl Med 1981;22:22-31

13. Druschky A, Hilz MJ, Platsch G, et al. Differentiation of Parkinson's disease and multiple system atrophy in early disease stages by means of I-123-MIBG-SPECT. J Neurol Sci 2000;175:3-12

14. Spiegel J, Hellwig D, Farmakis G, et al. Myocardial sympathetic degeneration correlates with clinical phenotype of Parkinson's disease. Mov Disord 2007;22:1004-08

15. Hoehn MM, Yahr MD. Parkinsonism: onset, progression and mortality. Neurology 1967;17:427-42

16. Imai $Y$, Hasegawa $K$. The revised Hasegawa's dementia scale (HDS-R) - evaluation of its usefulness as a screening test for dementia. J Hong Kong Coll Psychiatr 1994;4:20-24

17. Tanabe Y, Harada H, Sugihara S, et al. 123I-metaiodobenzylguani- 
dine myocardial scintigraphy in panic disorder. $\mathrm{J} \mathrm{Nucl} \mathrm{Med}$ 2004;45:1305-08

18. Tanaka M, Aihara Y, Ikeda S, et al. Neuromelanin-related contrast in the substantia nigra semiquantitatively evaluated by magnetic resonance imaging at 3T: comparison between normal aging and Parkinson disease. Clin Neurol 2011;51:14-20

19. Braak H, Del Tredici K, Rub U, et al. Staging of brain pathology related to sporadic Parkinson's disease. Neurobiol Aging 2003;24: 197-211

20. Halliday GM, Ophof A, Broe M, et al. $\boldsymbol{\alpha}$-Synuclein redistributes to neuromelanin lipid in the substantia nigra early in Parkinson's disease. Brain 2005;128:2654-64

21. Isaias IU, Marotta G, Pezzoli G, et al. Enhanced catecholamine transporter binding in the locus coeruleus of patients with early Parkinson disease. BMC Neurol 2011;11:88

22. Rommelfanger KS, Weinshenker D. Norepinephrine: the redheaded stepchild of Parkinson's disease. Biochem Pharmacol 2007;74: 177-90

23. Weinshenker D. Functional consequences of locus coeruleus degeneration in Alzheimer's disease. Curr Alzheimer Res 2008;5:342-45

24. Haneka MT, Nadrigny F, Regen T, et al. Locus ceruleus controls
Alzheimer's disease pathology by modulating microglial functions through norepinephrine. Proc Natl Acad Sci U S A 2010;107: 6058-63

25. Busch C, Bohl J, Ohm TG. Spatial, temporal and numeric analysis of Alzheimer changes in the nucleus ceruleus. Neurobiol Aging. 1997;18:401-06

26. Nakajima K, Yoshita M, Matsuo S, et al. Iodine-123-MIBG sympathetic imaging in Lewy-body diseases and related movement disorders. J Nucl Med Mol Imaging 2008;52:378-87

27. Okada Y, Ito Y, Aida J, et al. Lewy bodies in the sinoatrial nodal ganglion: clinicopathological studies. Pathol Int 2004;54:682-87

28. Orimo S, Takahashi A, Uchihara T, et al. Degeneration of cardiac sympathetic nerve begins in the early disease process of Parkinson's disease. Brain Pathol 2007;17:24-30

29. Rascol O, Schelosky L. ${ }^{123}$ I-metaiodobenzylguanidine scintigraphy in Parkinson's disease and related disorders. Mov Disord 2009; 24(Suppl 2):S732-41

30. Watanabe M, Takeda T, Nakamagoe K, et al. Sequential imaging analysis using MIBG scintigraphy revealed progressive degeneration of cardiac sympathetic nerve in Parkinson's disease. Eur J Neurol 2011;18:1010-13 